\title{
ABELIAN EXTENSIONS OF REGULAR LOCAL RINGS
}

\author{
PAUL ROBERTS
}

\begin{abstract}
ABSTRACr. The integral closure of a regular local ring in a finite Abelian extension of its quotient field is Cohen-Macaulay, provided that the degree of the extension is not divisible by the characteristic of the residue field.
\end{abstract}

TheOREM. Let $R$ be a regular local ring, $K$ its quotient field, and $L$ a finite Galois extension of $K$ with Abelian Galois group, which we denote $G$. Assume that the order of $G$ is not divisible by the characteristic of the residue field of $R$. Then if $S$ is the integral closure of $R$ in $L, S$ is Cohen-Macaulay.

Proof. We first note that since $L$ is assumed to be a Galois extension of $K$, and thus separable, $S$ is a finitely generated $R$-module. Since $S$ is integrally closed, it is therefore reflexive as an $R$-module; that is, the natural map from $S$ to $S^{* *}=$ $\operatorname{Hom}_{R}\left(\operatorname{Hom}_{R}(S, R), R\right)$ is an isomorphism (see Bourbaki [1, Chapter 7, §4.8]).

The action of the Galois group $G$ on $L$ makes $L$ into a $K[G]$-module; since any $K$-automorphism of $L$ must preserve the integral elements over $R, S$ similarly becomes an $R[G]$-module. The usual isomorphism: $S \otimes_{R} K \cong L$ can then be considered as an isomorphism of $K[G]$-modules.

The Normal Basis Theorem then says that there is an element of $L$ whose conjugates form a basis for $L$ over $K$; or, in other words, that $L$ is isomorphic to $K[G]$ as a $K[G]$-module. Hence $S$ is an $R[G]$-module such that $S \otimes_{R} K \cong K[G]$ as $K[G]$-module.

We wish to show that $S$ is a Cohen-Macaulay ring, and we will do this by showing that it is isomorphic to $R^{n}$ (where $n$ is the degree of $L$ over $K$ ) as an $R$-module. We first reduce to the case where $R$ is complete with algebraically closed residue field.

LEMMA. Let $R \rightarrow R^{\prime}$ be a faithfully flat extension of regular local rings. Then if $S^{\prime}=S \otimes_{R} R^{\prime}$ and $K^{\prime}$ is the quotient field of $R^{\prime}$, we have:

(a) $S^{\prime}$ is a reflexive $R^{\prime}$-module.

(b) If $S^{\prime} \simeq\left(R^{\prime}\right)^{n}$ as $R^{\prime}$-module, then $S \cong R^{n}$ as $R$-module.

(c) $S^{\prime}$ is an $R^{\prime}[G]$-module such that $S^{\prime} \otimes_{R^{\prime}} K^{\prime} \cong K^{\prime}[G]$ as $K^{\prime}[G]$-module.

Proof of Lemma. Since $R^{\prime}$ is flat over $R$, the natural map from $\operatorname{Hom}_{R}(M, N) \otimes_{R} R^{\prime}$ to $\operatorname{Hom}_{R^{\prime}}\left(R^{\prime} \otimes_{R} M, R^{\prime} \otimes_{R} N\right)$ is an isomorphism whenever $M$ is a finitely presented $R$-module; hence, applying this twice, $S^{* *} \otimes_{R} R^{\prime} \rightarrow$ $\left(S^{\prime}\right)^{* *}$ is an isomorphism. Thus the diagram

Received by the editors July 12, 1978 and, in revised form, November 30, 1978.

AMS (MOS) subject classifications (1970). Primary 13H10; Secondary 13B05.

(c) 1980 American Mathematical Society $0002-9939 / 80 / 0000-0102 / \$ 02.00$ 


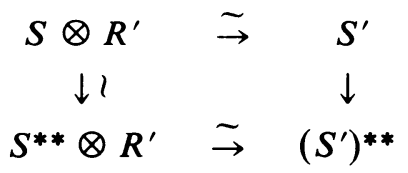

shows that $S^{\prime}$ is a reflexive $R^{\prime}$-module, proving (a).

To prove (b), we note that since $R^{\prime}$ is faithfully flat over $R$, tensoring with $R^{\prime}$ preserves minimal free resolutions. Hence if $S \otimes_{R} R^{\prime}$ is free over $R^{\prime}, S$ must be free over $R$.

Part (c) is obvious, since $S^{\prime} \otimes_{R^{\prime}} K^{\prime} \cong\left(S \otimes_{R} K\right) \otimes_{K} K^{\prime} \cong K^{\prime}[G]$.

From the Lemma we can assume that $R$ is complete; we can also assume that the residue field is algebraically closed (for this construction, see [2, Chapter $0, \S 6.8]$ ).

Let $k$ be the residue field of $R$. Since the order of $G$ is relatively prime to the characteristic of $k, G$ is Abelian, and $k$ is algebraically closed, $k[G]$ is isomorphic as a ring to a product of copies of $k$. Since $R$ is complete, this decomposition can be lifted to $R[G]$, and $R[G]$ is isomorphic as a ring to a product of copies of $R$. Write this decomposition:

$$
R[G] \cong R_{1} \times \ldots \times R_{n} ; \text { each } R_{i} \text { isomorphic to } R \text {. }
$$

Since $S$ is an $R[G]$-module, it must split also; we have:

$$
S \cong S_{1} \times \ldots \times S_{n} \text {. }
$$

We note that this decomposition is canonical, since each $S_{i}$ is uniquely defined as the set of elements of $S$ which are annihilated by all elements of $R_{1} \times \ldots \times R_{n}$ whose $i$ th component is zero.

Tensoring with $K$, we get

$$
\begin{gathered}
K[G] \cong R[G] \otimes_{R} K=\left(R_{1} \otimes K\right) \times \ldots \times\left(R_{n} \otimes K\right) \cong K \times \ldots \times K . \\
S \otimes_{R} K=\left(S_{1} \otimes K\right) \times \ldots \times\left(S_{n} \otimes K\right) .
\end{gathered}
$$

Since $S \otimes_{R} K \cong K[G]$ as $K[G]$-module and these decompositions are canonical, we must thus have $S_{i} \otimes_{R} K \cong R_{i} \otimes_{R} K \cong K$ for each $i$. Since $S$ is reflexive the same is true for each $S_{i}$; hence each $S_{i}$ is a reflexive $R$-module such that $S_{i} \otimes_{R} K \simeq K$, and is thus isomorphic to a divisorial ideal of $R$ (Bourbaki [1, Chapter 7, §4.2]). Since $R$ is a unique factorization domain, this implies that $S_{i} \simeq R$. Hence

$$
S \cong S_{1} \times \ldots \times S_{n} \cong R^{n} \text { as } R \text {-module. }
$$

Thus $S$ is Cohen-Macaulay, and this completes the proof of the theorem.

It can be seen from the proof of this theorem that if $R$ is assumed only to be integrally closed rather than regular, with the other hypotheses as above, then $S$ is a direct sum of divisorial ideals. In particular, if $R$ is a UFD, $S$ is free over $R$. We now give two examples to show that the other hypotheses cannot be weakened too much.

EXAMPle 1. This example, which was shown to me by M. Hochster, shows the necessity of the hypothesis on the characteristic of the residue field, at least for this proof of the theorem. 
Let $R$ be the ring $\hat{Z}_{2}[X, Y, U, V] /\left(Y^{2}-4 U-X^{2} V\right)$ localized at the maximal ideal $(Z, X, Y, U, V)$, where $\hat{Z}_{2}$ denotes the ring of 2 -adic integers. It can be verified that 2 generates a prime ideal in $R$, and if 2 is inverted the resulting ring is a localization of $\hat{Z}_{2}[X, Y, V]$ and is thus a UFD; hence by Nagata's criterion $R$ is a UFD. Let $K$ be the quotient field of $R$, let $L=K[\sqrt{V}]$, and let $S$ be the integral closure of $R$ in $L$. Then, letting $a=\frac{1}{2}(Y+X \sqrt{V})$, we have $a^{2}-Y a+U=0$, so $a$ is in $S$. We will show that not only is $S$ itself not free over $R$, but there are no nonzero $S$-modules which are free over $R$.

Suppose $F$ were such a module. Choosing an $R$-basis for $F$ then defines a ring homomorphism from $S$ to a ring of matrices with entries in $R$. Suppose $\sqrt{V}$ goes to the matrix $M$. Then $a$ goes to $\frac{1}{2}(Y \cdot I+X \cdot M)=N$, where $I$ is the identity matrix. Looking at a diagonal entry in this matrix gives an equation of the form $Y+X m=2 n$ for some $m$ and $n$ in $R$. But this implies that $Y$ is in the ideal of $R$ generated by $X$ and 2, which is not the case. Thus such a module $F$ cannot exist.

We remark that if $R$ were regular in this example, this would show that there were no Cohen-Macaulay modules over $S$ with support equal to $\operatorname{Spec}(S)$. For a discussion of this question we refer to M. Hochster [3].

EXAMPLE 2. In this example we show that the conclusion of the theorem is false if $G$ is assumed only to be solvable, or even nilpotent. We start with the ring $R_{1}=k[[X, Y, Z, W]] /(X Y-Z W)$, where $k$ is any field of characteristic zero. $R_{1}$ is an integrally closed domain and is a quadratic extension of the regular local ring $R=k[[X, Y, Z-W]]$; in fact, $R_{1}$ is clearly generated over $R$ by $Z+W$, and $(Z+W)^{2}=(Z-W)^{2}+4 X Y$ is in $R$.

Now let $a$ be any element of $R_{1}$ which is not a perfect square. Let $K_{1}$ be the quotient field of $R_{1}$, let $L^{\prime}=K_{1}[\sqrt{a}]$, and let $S^{\prime}$ be the integral closure of $R_{1}$ in $L^{\prime}$. If $L$ is the smallest Galois extension of $K$ containing $L^{\prime}$, then $L$ is generated over $L^{\prime}$ by a square root of the conjugate of $a$ over $K$, so the degree of $L$ over $K$ is either 4 or 8 , and $L$ is a nilpotent extension of $K$. Furthermore, if $S$ is the integral closure of $R$ in $L$, then $S^{\prime}$ is a direct summand of $S$ as an $R$-module, so that if $S^{\prime}$ is not free over $R$, neither is $S$. Thus it suffices to show that for suitable choice of $a$, the integral closure of $R_{1}$ in $K_{1}[\sqrt{a}]$ is not a free $R$-module.

Since $R_{1}$ is an integrally closed domain, the principal ideal $(a)$ is an intersection of symbolic powers of height one prime ideals:

$$
\text { (a) }=P_{1}^{\left(n_{1}\right)} \ldots P_{m}^{\left(n_{m}\right)} \text {. }
$$

An element $r+s \sqrt{a}$, with $r$ and $s$ in $K_{1}$, is integral over $R_{1}$ if and only if its trace $2 r$ and its norm $\left(r^{2}-a s^{2}\right)$ are in $R_{1}$; since 2 is a unit in $R_{1}$, this amounts to saying that $r \in R_{1}$ and $s^{2} a \in R_{1}$. Thus $S^{\prime}=R_{1}+I \sqrt{a}$, where $I=\left[s: s^{2} a \in R_{1}\right]$. Since an element $s$ of $K_{1}$ is in $R_{1}$ if and only if $s$ is in $\left(R_{1}\right)_{P}$ for all height one primes $P$, we have

$$
I=P_{1}^{\left(q_{1}\right)} \ldots P_{m}^{\left(q_{m}\right)}
$$

where $q_{i}=-\frac{1}{2} n_{i}$ if $n_{i}$ is even and $q_{i}=-\frac{1}{2} n_{i}+\frac{1}{2}$ if $n_{i}$ is odd.

Let $n$ be any positive integer, and let $b_{1}, \ldots, b_{2 n}$ be $2 n$ distinct nonzero elements of $k$. Let $P_{0}=(X, Z)$ and $P_{i}=\left(X-b_{i} Z, W-b_{i} Y\right)$ for $i=1,2, \ldots, 2 n$. Then 
the principal ideal $\left(X-b_{i} Z\right)$ is $P_{0} \cap P_{i}$ (to see this, divide by $X-b_{i} Z$; this gives $\left.k[[Y, Z, W]] /\left(\left(W-b_{i} Y\right) Z\right)\right)$. Let $a=\left(X-b_{1} Z\right)\left(X-b_{2} Z\right) \ldots\left(X-b_{2 n} Z\right)$. Then the principal ideal generated by $a$ is $P_{0}^{(2 n)} \cap P_{1} \cap \cdots \cap P_{2 n}$, and the corresponding fractional ideal $I$ is $P_{0}^{(-n)}$.

We wish to show that the minimal number of generators of $P_{0}^{(-n)}$ over $R_{1}$ is $n+1$; thus, if $n>1, P_{0}^{(-n)}$ cannot be free over $R$, since it would have to be free on 2 generators. We first multiply $P_{0}^{(-n)}$ by $X^{n}$; since $(X)=P_{0} \cap(X, W)$, this takes $P_{0}^{(-n)}$ to the isomorphic ideal $(X, W)^{(n)}$. Thus the result will follow if we can show that $(X, W)^{(n)}=(X, W)^{n}$ and that $X^{n}, X^{n-1} W, \ldots, W^{n}$ form a minimal set of generators for $(X, W)^{n}$. The second of these statements follows easily, since $X^{n}, X^{n-1} W, \ldots, W^{n}$ form a minimal set of generators for $(X, W)^{n}$ in $k[[X, Y, Z, W]] /(Y, Z) \cong k[[X, W]]$ and hence also in $R_{1}$.

To conclude that $(X, W)^{n}=(X, W)^{(n)}$, we must show that all zero-divisors of $\bar{R}=k[[X, Y, Z, W]] /\left(X^{n}, \ldots, W^{n}, X Y-Z W\right)$ are contained in $(X, W)$. If we invert $Y$, we can solve for $X$ :

$$
X=\frac{Z W}{Y}=\left(\frac{Z}{Y}\right) W, \quad \text { so }(X, W)^{n}=\left(W^{n}\right) .
$$

Thus we have

$$
\left[k[[X, Y, Z, W]] /\left(X^{n}, \ldots, W^{n}, X Y-Z W\right)\right]_{(Y)} \cong k[[Y, Z, W]]_{(Y)} /\left(W^{n}\right) .
$$

Since any zero-divisor in the latter ring is clearly contained in $(W)$, it will follow that the zero-divisors of $\bar{R}$ are in $(X, W)$ if we know that the map from $\bar{R}$ to $\bar{R}_{(Y)}$ is injective; that is, that $Y$ is not a zero-divisor.

Every element of $\bar{R}$ can be uniquely represented as a power series in the monomials $X^{i} Y^{j} Z^{k} W^{l}$ with $i+l<n$ and $k=0$ or $l=0$. Multiplication by $Y$ gives another nonzero power series with the same properties, so $Y$ is indeed a nonzero-divisor in $\bar{R}$.

Thus we have shown that there are Galois extensions of $R$ of degree 8 which are not Cohen-Macaulay, and in fact there are such extensions such that the minimal number of generators of the integral closure of $R$ as an $R$-module is arbitrarily large.

\section{REFERENCES}

1. N. Bourbaki, Commutative algebra, Hermann, Paris, 1972.

2. A. Grothendieck and J. Dieudonné, Eléments de géométrie algébrique. I, Springer-Verlag, Berlin and New York, 1971.

3. M. Hochster, Topics in the homological theory of modules over commutative rings, Regional Conference Series in Mathematics, No. 24, Amer. Math. Soc., Providence, R.I., 1975.

Department of Mathematics, University of Utah, Salt Lake City, Utah 84112 